\title{
Die gerechte Ordnung
}

Erhard Taverna
Drei Männer aus völlig verschiedenen Fachgebieten möchten Gerechtigkeit und Ordnung wieder herstellen. Der Soziologe fordert mehr Ordnung für benachteiligte Männer, der Kommissar muss die lädierte Ordnung wieder herstellen, der Bildungspolitiker die Schulpraxis in Ordnung bringen. Sie alle dienen der Gerechtigkeit, jeder auf seine Art.

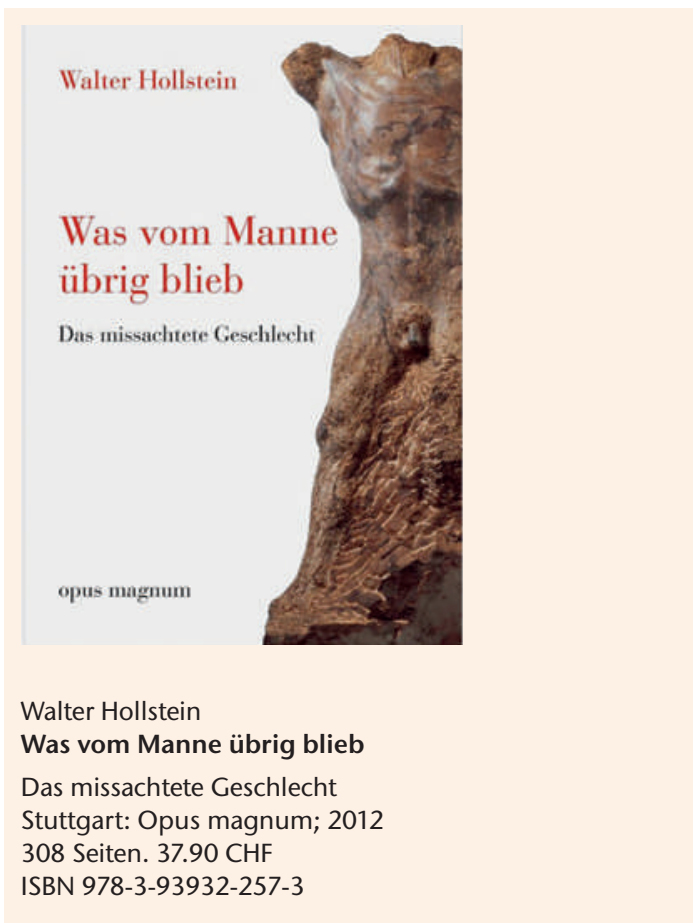

\section{Was vom Manne übrig blieb}

In Deutschland gibt es 54 Zentren für Frauen- und Geschlechterforschung an diversen Hochschulen, 40 Studiengänge und 132 Professuren, aber nicht einen einzigen Lehrstuhl für Männerforschung.

Mit dem Sachbuch «Was vom Manne übrig blieb» will Walter Hollstein, Professor für politische Soziologie und Gutachter des Europarates für Männerfragen, darlegen, was dringend politisch und pädagogisch geschehen muss, damit es der nachwachsenden männlichen Generation besser geht. Ein faktenreiches Buch, das in radikal-feministischen Kreisen auf heftige Ablehnung gestossen ist. $\mathrm{Zu}$ Unrecht. Denn was der Soziologe aufdeckt, ist keineswegs gegen den akademischen Feminismus gerichtet, wenn auch alarmierend und durch zahlreiche Studien belegt. Es war und ist sinnvoll, Männerherrschaft kritisch aufzuarbeiten, doch der dabei demontierte Begriff von Männlichkeit zeigt Nebenwirkungen in Schule, Recht, Medizin und Arbeit, die erst teilweise, zum Beispiel in einer gerechteren Regulierung des Sorgerechts, korrigiert werden. Orientierungslosigkeit und Identitätskonflikte fördern bei männlichen Erwachsenen und Jugendlichen Kriminalität, Suchtkarrieren, Krankheit, Verhaltensstörungen, Schulschwierigkeiten und Suizide.

Das Verdienst des Autors ist es, auf die vielfältigen Konfliktpotentiale hinzuweisen und deren Ursachen unmissverständlich und schonungslos zu benennen. Auch wo man Walter Hollsteins Folgerungen nicht immer bis ins Letzte folgen mag, weist sein völlig neu überarbeitetes Werk auf die blinden Flecken der aktuellen Genderforschung hin. Eine Broschüre des Schweizerischen Nationalfonds und des Eidgenössischen Büros für Gleichstellung von 2005 stellt fest, dass Gender Studies in der Schweiz mehrheitlich von Frauen betrieben werden. Es sei wünschenswert, dass der Männeranteil in Zukunft erheblich anwachse. Dem kann man nach dieser Lektüre nur zustimmen.

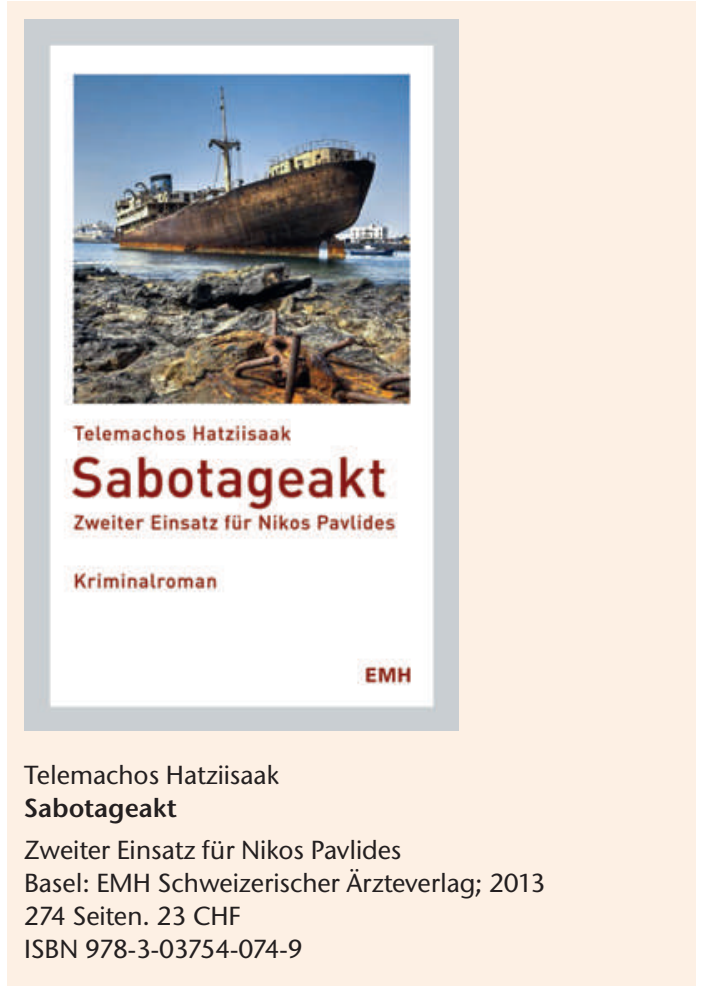

\section{Sabotageakt}

Telemachos Hatziisaak, Facharzt für Innere Medizin, lässt seinem Erstling «Kalte Allianz» einen zweiten Pavlides-Krimi folgen. Was 1994 in Thessaloniki mit einer gewaltigen Explosion im Hafen beginnt, endet 
2004 in den Archiven des griechischen Nachrichtendienstes, wo sich die letzten Puzzleteile eines blutigen Dramas zu einem Gesamtbild zusammenfügen. Eine spannend erzählte Geschichte, in der es um Waffenhandel, Korruption und politische Intrigen geht, und in der auch der Kommissar Nikos Pavlides nicht ganz ungeschoren davonkommt.

\section{Eine spannend erzählte Geschichte, in der auch}

\section{Kommissar Nikos Pavlides nicht ganz ungeschoren}

\section{davonkommt.}

Wieder versteht es der Autor, eine komplexe Handlung kunstvoll zu gliedern, zahlreiche Handlungsfäden logisch zu verknüpfen und dabei eine Spannung zu erzeugen, die vom Anfang bis zum Ende ungebrochen anhält. Dank seiner griechischen Herkunft kennt Hatziisaak die Schauplätze und Mentalitäten aus eigener Anschauung. Dieses Insiderwissen hat er jetzt erneut in einen Thriller verpackt, der Unterhaltung und Information auf hohem Niveau zusammenfügt. Ein Kunststück, das zahlreiche Leser und Leserinnen verdient.

\section{Philosophie einer humanen Bildung}

Von Jean-Jaques Rousseau über Wilhelm von Humboldt und Maria Montessori bis zu David Precht (Anna, die Schule und der liebe Gott, 2013) wird die Schule kritisiert und neu entworfen. Mehr noch als in den Medizindebatten spiegelt die aktuelle Bildungsdiskussion die Probleme einer fragmentierten, widersprüchlichen Gesellschaft. Kann die Schule allen Ansprüchen genügen? Sind jährlich $15 \%$ mehr Ritalinverordnungen in der Schweiz zu verantworten? Wird ein Notensystem unseren Kindern gerecht? Was verbindet Akademikerquoten und Jugendarbeitslosigkeit?

$\mathrm{Zu}$ all diesen Fragen gibt es eine wachsende Literaturfülle, die kaum jemand überblicken kann. Julian Nida-Rümelin, Ethiker, Philosoph und Kulturstaatsminister im Kabinett Schröder, besinnt sich auf die anthropologischen Konzepte einer humanen Bil-

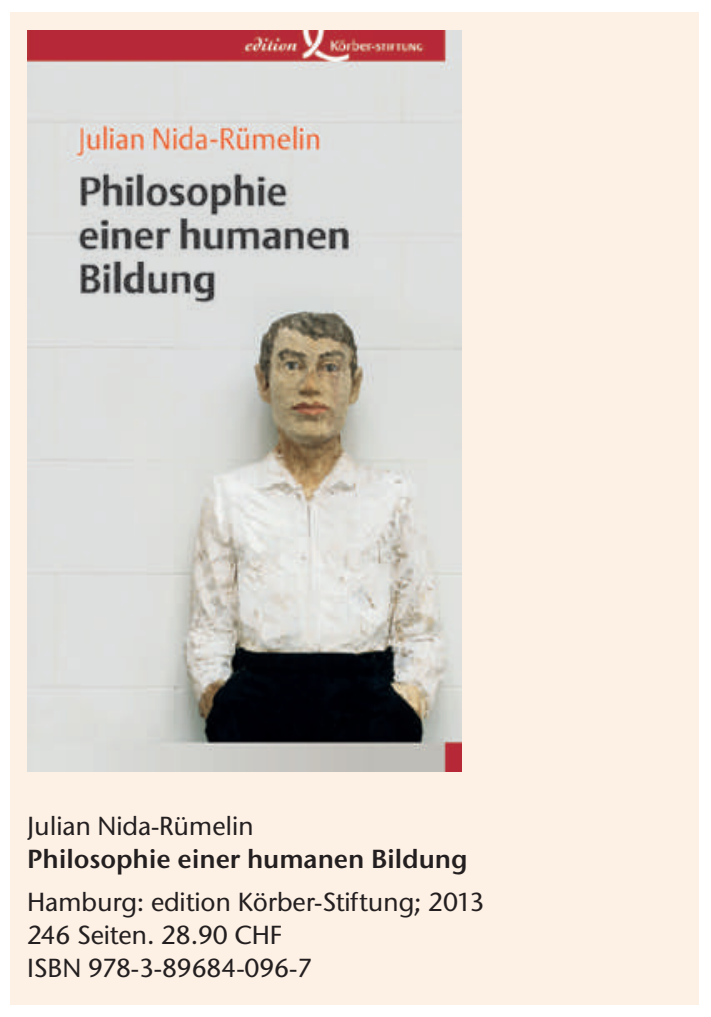

dung. Seine «Philosophie einer humanen Bildung» versteht sich als Orientierung. Sie soll den Blick öffnen für das, was Bildung bedeutet, denn die Bildungskrise sei nicht nur eine der Institutionen, sondern primär der Ideen. Vor allem im dritten Teil, «Bildungsziel: humane Praxis», decken sich seine programmatischen Ansichten mit denen vieler Reformpädagogen. Der allgemeinbildende Wissenserwerb soll der Persönlichkeitsbildung und nicht spezifischen beruflichen oder anderen Tätigkeiten dienen. «Die Bildungspraxis selektiert nicht und parzelliert nicht, sie geht von der Gleichwürdigkeit aller Menschen aus und fördert die Bedingungen eines gleichermassen gelungenen und autonomen Lebens.» Eltern und vor allem Medizinern, die sehr lange Zeit eine Schulbank gedrückt haben, hält Nida-Rümelin mit seiner einfach zu lesenden Schrift einen Spiegel oder vielleicht eine Wunschliste für ihre Kinder und Enkel vor. 